galaxies form through coalescence of smaller clouds, the pancakes of the adiabatic theory become natural reservoirs of internally supported low-mass gas clouds which form clusters of galaxies. Galaxies and clusters form at the same (relatively recent) epoch in this picture, with gaseous infall continuing into the present. This framework also allows gas-rich systems to survive until the present epoch without significant star formation. R. Terlevich (Institute of Astronomy, Cambridge) described observations of violent star formation in these HII galaxies, showing that the metallicity is correlated with the mass/light ratio, the effective temperature of the brightest stars and the total UV flux. These correlations are most plausibly explained as changes in the slope of the highmass star initial-mass function, in the sense that it is flatter at lower metallicities.

Like the HII galaxies, ellipticals appear simple single-population systems at first sight. Recent dynamical studies have, however, shown this to be far from true. C. Jenkins (RGO) showed that powerful radio galaxies appear to have significant rotation, along the minor axis, suggesting that these may be tri-axial systems. S. Phillips (University College, Cardiff), considering the radio properties of all Hubble types, showed a loose correlation between radio luminosity and surface brightness.

S. Lilly (University of Edinburgh) discussed infrared (IR) and optical observations of the more distant $3 C$ radio galaxies. While the IR colours are well fitted by passive evolution of a coeval population, the optical to IR colours required active star formation. However, the scatter in the latter indicates idiosyncratic variations from one galaxy to another, rather than the presence of a 'new' population. This is obviously important for population synthesis models, such as those described by $G$. Bruzual (University of Durham). By and large, ellipticals are well represented by models with a single burst of star formation, although an extant problem is the substantial UV rising branch observed in most such galaxies. M. Capaccioli (University of Padova) presented a review of IUE observations which show there to be little correlation between the UV flux and total luminosity. This flux, indicative of temperatures of $\sim 30,000 \mathrm{~K}$, is generally credited to horizontal branch stars or continuing star formation but, discussing the impact of binaries on population synthesis models, A. Collier (RGO) pointed out cataclysmic variables as another possible source. Finally, R. Ellis (University of Durham) suggested three areas that studies of galaxy evolution should focus on: an improved determination of the "standard" energy distribution of the different Hubble types; optical and IR studies of cluster galaxies, extending down the luminosity function and to higher $z$; and more extensive field redshift surveys, to $R-22$.

N. Reid is SERC Fellow at the Astronomy Centre, University of Sussex, Brighton BNI $9 R H$.

Mathematics

\title{
Novel techniques in bifurcation theory
}

from Ian Stewar!

BIFURCATION theory is the study of how solutions to differential equations can change as the values of numerical parameters vary. Traditionally a parameter value is a bifurcation point if the number of solutions (with given boundary conditions) changes there. For example, the equations describing an elastic rod under compression have a single solution (the unbuckled state) below a certain threshold of compression, and three solutions (buckle up, buckle down and an unstable unbuckled state) above threshold. Another important example is the Hopf bifurcation, in which a stable steady state becomes unstable and throws off a branch of oscillatory solutions. Many important natural phenomena have been modelled successfully by bifurcations of differential equations.

A major difficulty is to find conditions sufficient to guarantee bifurcation. The traditional approach has been heavily analytical, with the emphasis placed on delicate functional analysis. A forerunner of recent developments was the introduction of topological methods, notably degree theory, by Krasnoselskii ${ }^{1}$ in 1964 . In the last five years several novel and powerful techniques have been imported from pure mathematics, and it is likely that these will produce fundamental changes in the way bifurcation problems are tackled in the future.

Singularity theory, an offshoot of René Thom's catastrophe theory, appears in the generic bifurcation theory of Chow, Hale and Mallet-Paret ${ }^{2}$. A related and highly significant development is the imperfect bifurcation theory of Golubitsky and Schaeffer ${ }^{3}$, which adapts ideas from catastrophe theory to the analysis of bifurcations. The result is a rigorous method for detecting and classifying bifurcations of steady states, together with a sophisticated technique, unfolding theory, for analysing perturbations. The results generalize to the important case where symmetries are acting ${ }^{4}$, and to oscillatory systems exhibiting a degenerate version of the Hopf bifurcation $^{5}$. Applications include an explanation of mode-jumping in the buckling of a rectangular plate ${ }^{6}$, the explosion peninsula in chemical reactions ${ }^{7}$, the behaviour of Bénard convection cells in the spherical annulus ${ }^{8}$ and the plane ${ }^{9}$, and periodic wavetrains in the HodgkinHuxley nerve impulse equations ${ }^{10}$.

Topological dynamics, the creation of Stephen Smale and his co-workers, is increasingly being applied to bifurcations. Smale $^{11}$ generalized the definition to allow changes in the topological type (and not just the number) of solutions, a farreaching idea now bearing fruit in the theory of chaotic systems. These are deterministic systems that exhibit what appears to be random behaviour. Important contributions have also been made by the Russian school of Vladimir Arnol'd.

The latest import from modern pure mathematics is algebraic geometry. A recent paper of Buchner, Marsden and Schechter ${ }^{12}$ proves a very general (and computable) sufficient condition for bifurcation, which extends several of the standard theorems. Their argument makes essential use of the 'blowing-up' construction developed by algebraic geometers to resolve (get rid of) singularities (bad points) in algebraic varieties (solution sets of polynomial equations). A simple version of blowing-up is to replace a cone by a cylinder, by expanding the vertex point into a circle. The modern version is highly abstract and a good deal more sophisticated, and was developed as a technical tool within pure mathematics. The results of Buchner et al. apply to a wide range of bifurcation problems, including multi-parameter problems and multiple eigenvalue problems, and reveal the importance of a certain regularity condition on the leading terms of the equation. Several extensions and applications are promised.

Pure mathematics is sometimes criticized for a lack of attention to application. While it is no doubt true that individual mathematicians do not always bear application in mind, it appears that the results obtained by them may nonetheless prove useful in applied science. Powerful mathematical techniques remain powerful, no matter what their origins may be; and the origins in no way determine the areas to which they may be applied.

Ian Stewart is currently at the Department of Mathematics, University of Housion, Central Campus, Houston, Texas 7704.

1. Krasnoselskij, V. M. Topological Methods in the Theory of Nonlinear Integral Equations (Pergamon, Oxford, 1964).

Chow, S.-N, Hale, J.K, \& Mallet-Paret, I. Arch. Rat Mech. Anal. 59, I59(1975); 62, 209 (1976).

Mech. Anal. 59, I59(1975); 62, $209(1976)$.
3. Golubilsky, M. \& Schaeffer, D. Commun. pure appl. Math. 32, 21 (1979).

4. Golubitsky, M. \& Schaeffer, D. Commun. Math. Phys. 67, 205 (1979).

5. Golubitsky, M. \& Langford, W. J. diff, Eq. 41, 375 (1981). 6. Schaeffer, D. \& Golubilsky, M. Commun. Math. Phys. 69, 209 (1979).

209 (1979)

(1) Keyfitz, B.L. \& Schaeffer, D. Commun. pure appl. Math. 34, 433 (1981).

8. Golubilsky, M. \& Schaeffer, D. Commun. pure appl. Math. 35, 81 (1982).

9. Buzano, E. \& Golubilsky, M. Phil. Trans. R. Soc. 308.617 (1983).

10. Labouriau, 1. Ihesis, Univ. Warwick (in preparation)

11. Smale, S. Invent, Math. 10, 305 (1970)

12. Buchner, M., Marsden, J. \& Schechter, S. J. diff. Eq. 48. 404 (1983). 